\title{
Usages de l'internet par les personnes âgées en Turquie
}

The elderly internet uses in Turkey

Uso de internet por mayor en Turquía

\section{Nazlı Ülbay Aytuna}

\section{OpenEdition}

\section{Journals}

Édition électronique

URL : http://journals.openedition.org/ctd/532

DOI : $10.4000 /$ ctd. 532

ISSN : 2491-1437

Éditeur

Chaire Unesco Pratiques émergentes en technologies et communication pour le développement

Référence électronique

Nazlı Ülbay Aytuna, «Usages de l'internet par les personnes âgées en Turquie », Communication, technologies et développement [En ligne], 6 | 2018, mis en ligne le 18 décembre 2018, consulté le 20 avril 2019. URL : http://journals.openedition.org/ctd/532 ; DOI : 10.4000/ctd.532

Ce document a été généré automatiquement le 20 avril 2019.

Communication, technologies et développement 


\title{
Usages de l'internet par les personnes âgées en Turquie
}

\author{
The elderly internet uses in Turkey \\ Uso de internet por mayor en Turquía
}

Nazlı Ülbay Aytuna

\section{NOTE DE L'AUTEUR}

Cet article a été soutenu financièrement par Le Fonds de Recherche de l'Universite Galatasaray no : 17.300 .004

1 Dès le début des années 1990, nombre de travaux insistent sur l'exclusion de certains groupes sociaux par rapport aux nouvelles technologies. En effet, les études des usages développés à partir des années 2000 visent à explorer le sens donné aux technologies à travers l'observation des pratiques et des interactions sociales (DiMaggio et Hargittai, 2002 ; Lelong, 2003, Van Deursen, VanDijk, 2014, Lobet-Maris, 2011). La fracture numérique ainsi définie représente davantage qu'une simple question d'accès. Il s'agit en effet d'un phénomène sociologique qui reflète les inégalités sociales, économiques, culturelles et d'apprentissage de manière plus large. De fait, « La société de l'information se construit sur fond de clivage, de coupure, de cassure, de déchirure, de séparation entre ceux qui en font déjà partie et ceux qui ne sauraient s'y inscrire." (Kiyindou, 2007 : p. 1). Norris (2001) ajoute que les personnes âgées se trouvent dans le «trou noir de l'information » de Castells (2001) en faisant valoir que la différence générationnelle est une caractéristique clé de l'adaptation à Internet. La conception de la fracture est également considérée comme une question de compétence et de satisfaction. Même si les fractures ont diminué en termes d'accès (Katz et al. 2001, Smolenski, 2000), des fractures importantes se montrent en termes de modes d'utilisation et de bénéfices obtenus (Lazarus et Mora, 2000 ; Norris, 2001). Celles-ci demeurent un problème persistant en termes d'âge, car les personnes âgées restent la catégorie la plus déconnectée du monde virtuel. 
2 La Turquie, à l'image d'autres pays européens, devra faire face, dans un avenir proche, à un accroissement de sa population âgée. En effet, ce sont les plus de 65 ans qui connaissent l'expansion la plus rapide sur le plan démographique au niveau national. Selon l'Institut Statistique de Turquie ${ }^{1}$, la population âgée, qui dénombrait 5.668.003 individus $(7,5 \%)$ en 2012, est montée à 6.651 .503 individus $(8,3 \%)$ en 2016. De plus, selon ce même rapport, on estime qu'en 2023, la part de la population âgée représenterait $21 \%$ de la population totale turque. Cette population croissante fait face à des barrières qui l'empêchent de s'adapter aux besoins de la société de l'information et au numérique. Par ailleurs, les références statistiques de l'usage de l'internet selon les différentes catégories de l'âge nous permettent de prévoir cette fracture numérique : $84,3 \%$ des jeunes âgés de 16 à 24 ans déclarent avoir utilisé un ordinateur ou Internet au cours des 3 derniers mois ${ }^{2}$, contre seulement $8,8 \%$ de la population de 65 ans et plus. En outre, cette fracture numérique devient encore plus spectaculaire lorsqu'on prend en compte les différences de genre. Seulement $4,3 \%$ de la population féminine de plus de 65 ans affirme avoir fait usage d'Internet au cours des trois derniers mois. Prenant en considération les difficultés d'intégrer la population âgée à l'usage actif de l'Internet, le travail vise à souligner le risque de négligence d'une partie importante de la population dans la société d'information.

3 En effet, il existe plusieurs études qui analysent l'utilisation d'Internet par les personnes âgées. Morris et Goodman (2007), par exemple, se penchent sur les activités informatiques et Internet, et les raisons de son utilisation ou de sa non-utilisation dans le Derbyshire (en Angleterre) et en Écosse. Helsper (2009) explore, quant à lui, les facteurs qui échappent au contrôle direct d'une personne et qui limitent leur accès et leur capacité à utiliser les nouvelles technologies de l'information et de la communication en s'attachant au concept d'“exclusion numérique". Hilt et Lischultz (2004) mènent des entretiens en profondeur avec des utilisateurs informatiques âgés originaires des États du Midwest aux États-Unis dans l'optique d'observer un large éventail d'intérêt et d'utilisation. Zuniga et al. (2003) insistent, eux, sur la fracture numérique en observant les différentes dimensions de l'utilisation des outils numériques et de la gratification qui en découle. En Chine, une étude met en avant les prédicateurs de l'utilisation d'Internet afin de rechercher des informations sur la santé en ligne des personnes âgées (Wong, al. 2014). En Israël, une étude compare 10 participants plus âgés dans des sessions de formation en informatique avec dix non participants pour obtenir des points de vue sur l'utilisation d'ordinateurs et d'Internet (Blit-Cohen, Litwin, 2004). En Turquie, Tekedere et Arpac1 effectuent une recherche quantitative dans l'objectif de révéler les préférences d'utilisation de l'internet d'un groupe de 106 personnes âgées vivant à Ankara (Tekedere, Arpac1, 2016: 378). Une autre étude intéressante établit une comparaison entre la dépendance à Internet des personnes âgées vivant au Kosovo et ceux en Turquie (Abac1 et al. 2013)

4 La présente étude consiste à mettre en perspective l'usage de l'Internet par les personnes âgées et à en débattre à partir d'une enquête qualitative réalisée en Turquie. Les entretiens effectués s'inscrivent dans l'approche théorique des usages et des gratifications (Uses and gratifications theory) qui porte sur la manière dont les gens utilisent les médias. Selon cette perspective, ces derniers font usage des différents médias à des fins variées et choisissent notamment les médias en fonction de leurs besoins ou de leurs objectifs spécifiques ${ }^{3}$. Par conséquent, plutôt que de se demander comment l'utilisation des médias influence les utilisateurs, cette approche s'interroge sur la 
manière dont les besoins fondamentaux des utilisateurs influencent les choix des médias. Ainsi, la présente recherche s'intéresse plus particulièrement aux besoins et aux usages que les personnes âgées font de l'Internet et aux gratifications qu'ils en retirent. Que font les personnes âgées avec l'Internet? Quelles sont leurs motivations et les raisons de leurs habitudes? À noter également que la question de la recherche ne concerne pas seulement les motivations de l'usage, mais aussi celles du non-usage.

\section{Méthodologie d'analyse}

5 La recherche repose sur des entretiens semi-directifs réalisés auprès de 36 personnes âgées (de plus de 65 ans) vivant à Istanbul. Le plus jeune participant était âgé de 65 ans, le plus âgé en avait 81. La population étudiée est composée de 20 utilisateurs et de 16 nonutilisateurs. Les personnes âgées non-utilisateurs comprenaient à la fois des personnes qui n'avaient jamais utilisé Internet auparavant et celles qui en avaient fait l'expérience, mais qui avaient cessé de l'utiliser. Les utilisateurs ont été choisis parmi les participants aux cours de formation en informatique dans les centres d'éducation publique ${ }^{4}$. Les caractéristiques démographiques de ces centres dépendent en général de leur emplacement. Les centres éloignés du centre-ville reflètent un niveau socio-économique plus faible, ce qui impacte également l'âge moyen des participants. Il est d'ailleurs à noter que la plupart des utilisateurs interrogés dans ces centres ont un niveau d'études élevé et vivent dans le centre-ville.

6 La recherche a eu lieu au cours de la période allant de novembre 2016 à mars 2017. Chaque entrevue a duré environ une demi-heure. En premier lieu, on a posé des questions générales aux sondés, telles que la façon dont elles passent habituellement leur journée ou ce qu'elles aiment faire le week-end. Dans un second temps, on les a interrogés sur leur consommation des médias en général. Enfin, on a terminé avec des questions sur l'Internet, les habitudes d'usage de l'ordinateur et les réseaux sociaux ${ }^{5}$.

\section{Les motivations des utilisateurs}

7 La présente étude confirme les résultats des recherches précédentes en démontrant que les personnes âgées utilisent l'Internet d'une manière plus restreinte par rapport aux autres groupes d'âge. Les principales motivations des utilisateurs se révèlent être « rechercher des choses» ou maintenir la «connectivité sociale» existante. En effet, presque tous les interviewés étaient des utilisateurs actifs des médias conventionnels. Internet a modifié leurs manières d'aborder et de consommer les médias. Par exemple, la lecture des journaux en ligne est une activité commune des personnes âgées. De plus, jouer à des jeux; écouter de la musique sont également mentionnés parmi leurs principales activités sur Internet. Les utilisateurs affirment plutôt avoir des attitudes positives envers Internet, comme le sentiment d'être jeune et d'être confortable, la sensation de supériorité, d'amusement et de confiance (tableau 1).

\section{Rechercher des choses}

8 Pour les utilisateurs, Internet est un moyen efficace d'accéder à l'information. Les personnes âgées utilisent en général les moteurs de recherche pour rechercher des sujets 
spécifiques selon leurs intérêts, pour suivre des nouvelles sur les événements actuels, pour rechercher des idées de vacances, pour organiser leurs voyages et pour examiner les informations météorologiques. Par contre, la recherche d'informations sur la santé a été rarement mentionnée.

«J'aime rechercher des trucs. Par exemple, je recherche des poèmes, mais pas une recherche approfondie bien sûr. Je n'ai pas beaucoup de temps, parce que mon mari est tellement malade. » (E., interviewée femme, 72 ans, ingénieure retraitée)

«Maintenant je consulte directement sur Google quand j'ai quelque chose dans ma tête, avant de regarder l'encyclopédie. » (F., interviewé homme, 81 ans, employé de l'université retraité)

«Avant l'internet, il n'y avait que le téléphone à la maison. Si vous n'êtes pas à la maison, vous ne recevrez aucune nouvelle. Pour l'instant, je sais ce qui se passe dans le monde entier.» (T., interviewée femme, 70 ans, pas d'expérience professionnelle)

\section{Connectivité sociale}

Pour les personnes âgées, qui ont des problèmes de mobilité ou qui vivent géographiquement loin de leur famille, l'Internet présent des opportunités de socialisation et d'établissement du soutien social et familial.

«J'ai commencé à utiliser Internet après que mon fils ait déménagé en Allemagne. Normalement, les appels téléphoniques entre les deux pays sont très coûteux; Je ne peux pas appeler mon fils tous les jours. Grâce à Internet, nous pouvons rester en contact. L'utilisation d'Internet est donc importante pour moi. Sans ça, je ne pouvais pas voir et recevoir des nouvelles de mon fils. Chaque jour, il m'envoie une photo. » (N., interviewée femme, 65 ans, pas d'expérience professionnelle.)

"J'ai beaucoup d'amis dans Face (Facebook), avec qui je suis toujours en contact. Quand quelqu'un est mort ou un bébé est né, nous nous communiquons les uns avec les autres. » (Y., interviewé homme, 73 ans, professeur d'histoire retraité)

«J'ai trouvé tous mes amis de lycée sur Facebook. Je suis heureuse de rester en contact avec mes anciens amis. Nous ne pouvons pas nous voir tout le temps, mais je me sens heureuse quand nous restons en contact. C'est vraiment génial, mais je pense que je ne peux pas suivre la technologie. » (T., interviewée femme, 65 ans, assistante-exécutif retraitée)

Tableau 1 : Motivation des Utilisateurs

\begin{tabular}{|l|}
\hline Activités \\
\hline Faire des recherches (cuisine, littérature, religion, voyages, artisanat) \\
\hline Lire le journal quotidien \\
\hline Se connecter avec les amis et avec les membres de la famille \\
\hline Jouer des jeux \\
\hline Écouter de la musique \\
\hline Attitudes \\
\hline Supériorité \\
\hline
\end{tabular}


Amusement (Temps de loisir)

Se sentir à l'aise

Fraicheur (se sentir plus jeune) Confort

\section{Limitations des utilisateurs}

Les interviewés ont très souvent déclaré des difficultés et des limitations rencontrées dans leur accès et utilisation de l'Internet. Le manque de confiance en soi est l'une des principales préoccupations. En général, les personnes âgées ne pensent pas que l'internet est destiné à leur usage. Autrement dit, la croyance en leur incapacité à devenir un expert en informatique est globalement partagée par les sondés. Les problèmes de protection de la vie privée et le manque d'intérêt sont d'autres obstacles majeurs pour les utilisateurs âgés.

« Mes amis qui utilisent Facebook et Instagram sont plus jeunes que moi. Mais ils ne m'excluent jamais à cause de mon âge ; ils sont tellement surpris que je l'utilise à cet âge. « (À., interviewé homme, 75 ans, médecin retraité)

"C'est compliqué pour moi. Je pose les mêmes questions à plusieurs reprises. »(M., interviewé homme, 81 ans, employé de l'université retraité)

«C'est cool! J'aimerais être jeune et grandir avec Internet. «(H., interviewé homme, 67 ans, interviewé employé du secteur privé retraité)

« Il y a quelques années, mon fils m'a apporté un ordinateur. Une fois, j’ai essayé de l'ouvrir, mais il a verrouillé, je n'ai pas compris ce qui s'est passé. J'ai eu peur. Ensuite, mon fils est venu à la maison et a commencé à me crier à cause d'un ordinateur verrouillé. Ensuite, j'ai décidé de commencer à un cours informatique. «(À., interviewé homme,81 ans, médecin retraité)

\section{Interactivité limitée}

L'interactivité a été définie comme la mesure dans laquelle les participants au processus de communication ont le contrôle et peuvent échanger des rôles dans leurs discours mutuels (Williams et al., 1988 : 10). Les utilisateurs âgés s'engagent seulement dans une petite gamme d'activités et s'affichent un intérêt limité pour les fonctions interactives d'Internet. L'interaction sociale qui comprend à la fois les échanges d'information entre les personnes et les groupes en ligne est ainsi restreinte. De même, les utilisateurs âgés ne souhaitent pas exposer leur vie privée en ligne. Même si les personnes âgées sont bien formées, saines et socialement actives, elles utilisent Internet de façon limitée. Parfois, cette limitation est également causée par les membres de la famille. Les personnes interrogées prononcent généralement des inquiétudes en matière de protection de la vie privée.

«Parfois, je veux exprimer certaines de mes opinions politiques, mais mon fils me dit ne pas le faire "

(M., interviewé homme, 81 ans, employé de l'université retraité)

"Je suis avocat, j'étais un » dinosaure " jusqu'à récemment, et je n'ai même pas touché le clavier de l'ordinateur. Maintenant, j'apprends un peu. Mais je n'envoie pas de documents sur Internet. Je ne l'envoie pas parce que je ne peux pas faire 
confiance au système. En cas d'interruption, cela peut causer un problème juridique. » (H., interviewé homme, 69 ans, avocat)

«Contrairement aux jeunes, j'évite de partager ma vie privée sur Facebook. Je ne partage que des informations utiles. » (S., interviewée femme, 65 ans, professeur d'anglais retraité).

\section{Manque d'intérêt}

12 Les personnes âgées croient qu'elles ne sont pas capables de contrôler ou de gérer les nouvelles technologies. Principalement, le manque d'intérêt est causé par les croyances, comme les ordinateurs ne sont pas appropriés ou trop compliqués pour les personnes âgées. Autrement, le manque d'intérêt peut s'expliquer aussi par le manque de connaissance puisqu'ils ne savaient pas ce qu'ils pouvaient faire en l'utilisant. Certains travaux démontrent que l'utilité perçue est en relation avec la qualité de l'information diffusée sur Internet ainsi qu'avec l'encouragement des membres de la famille et des amis (Morell et a.l., 2000; Trocchia et a.l., 2000). Ainsi, la facilité d'utilisation perçue de l'Internet affecte son utilisation en affectant les sentiments d'autocompétence de l'utilisateur (Teo,1999).

«Ma fille m'a offert un ordinateur il y a quelques années. Mais je n'étais pas très intéressée, mon mari me demandait de chercher des informations médicales, je regardais des choses comme ça. Mais maintenant, je peux pratiquer plusieurs choses que j'ai apprises, alors je m'intéresse.» (M., interviewée femme, 79 ans, comptable retraitée)

"Ça ne m'intéresse pas du tout d'écouter de la musique ou regarder un film sur Internet. Je n'ai pas le temps, car je suis plus proche de la mort maintenant, je dois évaluer le temps le plus possible. » (À., interviewé homme, 75 ans, médecin retraité) "Je n'ai pas d'accès Internet à ma maison; Je n'ai pas besoin du tout. J'utilise l'ordinateur / internet pendant des cours d'informatique, mais je pense que je dois fournir l'accès à Internet à la maison. Celui qui vient à ma maison demande d'abord s'il y a Internet ou non.» (H., interviewée femme, 67 ans, pas d'expérience professionnelle)

\section{Les motivations des non-utilisateurs}

Le plus souvent, les personnes âgées non-utilisatrices sont décrites en termes de particularités sociodémographiques telles que le genre, l'éducation et la composition du ménage (Helsper et Reisdorf, 2013, Morris et al., 2007). Pour expliquer les désengagements des personnes âgées, certains travaux sur l'usage de l'Internet insistent sur des raisons précises, comme manque d'expérience, manque de compétence, et des coûts de connexion élevés (Helsper et Reisdorf, 2013 ; Lee et al., 2011; Millward, 2003 ; Morris et al., 2007, Peacock et Künemund, 2007). Les caractéristiques démographiques ou les handicaps physiques tels que la faible vision, les déficiences cognitives et les limitations des compétences motrices sont principalement considérés comme responsables. Surtout en ce qui le cas de la Turquie, l'insuffisance de l'éducation est un autre problème majeur'.

14 Le présent travail révèle trois groupes de non-utisateurs : Le premier groupe concerne des non-utilisateurs radicaux. Il s'agit de personnes âgées qui s'opposent à l'usage supposant que l'internet est responsable de tout le malheur dans la société. Le deuxième groupe souligne les difficultés d'utilisation et le manque de compétences lié à leur condition physique et mentale. Pour le troisième, l'internet n'est pas nécessaire, voire inutile. 
«Internet ne changera rien. L'un trouve une fille, l'autre trouve un mari ... Oui, cela s'est produit dans mon village, elle a trouvé un mari sur Internet ... à mon âge, dans notre village, que faire de l'Internet. » (M., femme, âgée de 77 ans, analphabète)

«Depuis l'entrée d'Internet dans nos vies, la violence à l'égard des femmes a augmenté ... L'État doit restreindre l'utilisation ... En tout cas, il est trop tard pour utiliser Internet, nous ne pouvons pas voir sans porter de lunettes » (T., homme, âge 73, diplômé de l'école élémentaire, l'ouvrier à l'usine retraité).

"Que puis-je faire sur Internet à mon âge ? Je ne suis pas capable d'utiliser Internet, mais j'ai appris à écouter le Coran sur YouTube en utilisant mon Smartphone. Et je peux appeler quelqu'un, c'est tout. » (M., femme, âgée de 77 ans, analphabète).

"J'aimerais utiliser, mais à mon âge, je ne veux plus, j'aimerais si j'avais appris à temps. » (E., femme, 81 ans, pas de grade scolaire)

Tableau 2 : Motivations des non-utilisateurs

\begin{tabular}{l} 
Représentation de l'internet \\
Source de violence Source de malaise sociale Non utile \\
Pour les jeunes \\
\hline Raisons de désengagement Manque du temps Manque d'intérêt \\
Manque de confiance en soi \\
Manque de compétence physique et mentale Analphabétisation
\end{tabular}

\section{Conclusions}

15 La présente étude, fondée sur des entretiens réalisés en Turquie, confirme les résultats d'études antérieures effectuées dans différents contextes socioculturels. Elle confirme le fait que la population âgée montre un faible ajustement au développement technologique y compris l'usage de l'Internet. Dans le cas d'une comparaison avec des jeunes générations, l'écart d'usage devient plus révélateur. L'étude que nous avons menée sur l'utilisation de l'Internet par les enfants en Turquie indiquait également cette fracture numérique en insistant sur l'usage excessif des enfants (Aytuna et al., 2014).

16 La croissance rapide de cette population âgée et le potentiel d'Internet proposé pour surmonter leur isolement social, pour accéder à l'information utile et pour développer et maintenir leur indépendance en fait un sujet important à aborder. En ce sens, les formations spécifiques des personnes âgées à la technologie informatique sont indispensables pour réduire les obstacles potentiels et augmenter la perception par les personnes âgées quant à la facilité de l'utilisation de l'outil informatique. Ces dernières peuvent également encourager les utilisateurs plus âgés à entreprendre des activités plus variées sur Internet. 


\section{BIBLIOGRAPHIE}

Alain, Kiyindou. « De la fracture numérique à la fracture cognitive : pour une nouvelle approche de la société de l'information. » Thémat'IC, 2007, http://www.enssib.fr/bibliotheque-numerique/ documents/1948-de-la-fracture-numerique-a-la-fracture-cognitive-pour-une-nouvelleapproche-de-la-societe-de-l-information.pdf.

Alain Rallet, Fabrice Rochelandet, « La fracture numérique : une faille sans fondement ? », Réseaux, 2004/5 (n 127-128) : 19-54.

Alexander Van Deursen, Jan Van Dijk. "The digital divide shifts to differences in usage." New media \& society 16.3 (2014) : 507-526.

Anne Morris, Joy Goodman, Helena Brading. "Internet use and non-use : views of older users", Universal Access in the Information Society 6.1 (2007) : 43-57.

Antonio González, M. Paz Ramírez, and Vicente Viadel, “Attitudes of the elderly toward information and communications technologies", Educational Gerontology 38.9 (2012) : 585-594.

Antonio González, María Paz Ramírez,Vicente Viadel, "ICT learning by older adults and their attitudes toward computer use", Current gerontology and geriatrics research 2015 (2015).

Bob Lee, Yiwei Chen, and Lynne Hewitt, “Age differences in constraints encountered by seniors in their use of computers and the internet", Computers in Human Behavior 27.3 (2011) : 1231-1237.

Claire Lobet-Maris, “Âge et usages informatiques”, Communications 1 (2011) : 19-28.

Edith Blit-Cohen, Howard Litwin "Elder participation in cyberspace : A qualitative analysis of Israeli retirees", Journal of Aging Studies 18.4 (2004) : 385-398.

Ellen Helsper, “The ageing internet : digital choice and exclusion among the elderly”, Working with older people 13.4 (2009) : 28-33.

Ellen Helsper, Bianca C. Reisdorf, "A quantitative examination of explanations for reasons for internet nonuse", Cyberpsychology, Behavior, and Social Networking 16.2 (2013) : 94-99.

Elihu Katz, Jay G. Blumler, Michael Gurevitch, "Uses and gratifications research", The Public Opinion Quarterly, 37.4 (1973) : 509-523.

Frederick Williams, Ronald E. Rice, Everett M. Rogers, Research methods and the new media, Simon and Schuster, 1988.

Hakan Tekedere, Fatma Arpacı, "Orta yaş ve yaşlı bireylerin internet ve sosyal medyaya yönelik görüşleri”, Türkiye Sosyal Araştırmalar Dergisi 2 (2016) : 377-392.

Härkönen, A. Mattila, M. and Munnukka, J., “Technology usage among ageing consumers”, 2002, https://www.mit.jyu.fi/agora-center/inbct/InBCT42/ageing_and_technology.html Erişim Tarihi : 11.10 .2016

Jacqueline K. Eastman, Rajesh Iyer, “The elderly's uses and attitudes towards the Internet”, Journal of Consumer Marketing 21.3 (2004) : 208-220.

Jaeho Cho, et al., "Beyond access : The digital divide and Internet uses and gratifications", It \& Society 1.4 (2003) : 46-72. 
Kerryellen G.Vroman, Sajay Arthanat, Catherine Lysack, "Who over 65 is online ?" Older adults' dispositions toward information communication technology", Computers in Human Behavior 43 (2015) : 156-166.

Manuel Castells, La galaxie internet, Plaza y Janes, Madrid, 2001.

Metin Özkul, Ișıl KALAYCI, “Türk,ye'de Yaşlılık Çalıșmaları”, Sosyoloji Konferansları 52 (2015).

Michael L.Hilt, Jeremy H. Lipschultz, "Elderly Americans and the Internet : E-mail, TV news, information and entertainment websites", Educational Gerontology 30.1 (2004) : 57-72.

Milan Kubiatko, et al., "The using of and attitudes toward Internet and information and communication technologies in different age groups", Journal of Current Issues in Media \& Telecommunications 7.3 (2015).

Nazlı Aytuna, Doğu Toksöz, Neşe Öztemir, Dijital yaşayan çocuklar ? Çocukların bilgisayar ve internet kullanım (Les enfants qui vivent dans le digital : usages de l'internet/ordinateur par les enfants) éditions de l'Établissement de Radio et Télévision de Turquie (TRT) Ankara 2014 pp. 149-172.

Oya Hazer, Vildan Sökmen K1lınç, « Kırkbeş yaş ve üzeri yetişkinlerin günlük yaşamda teknolojiyi kullanma durumlarının incelenmesi », Geriatri Dergisi, 12.2 (2009) : 82-92.

Pau DiMaggio, Eszter Hargittai, “The new digital inequality : Social stratification among Internet users", American Sociological Association annual meetings, Chicago, 2002.

Peter Millward, “The'grey digital divide' : Perception, exclusion and barriers of access to the Internet for older people", First Monday 8.7 (2003).

Philip Palmgreen, "Uses and gratifications : A theoretical perspective", Annals of the International Communication Association 8.1 (1984) : 20-55.

Philip J. Trocchia, Swinder Janda, “A phenomenological investigation of Internet usage among older individuals”, Journal of consumer marketing 17.7 (2000) : 605-616.

Pippa Norris, Digital divide : Civic engagement, information poverty, and the Internet worldwide. Cambridge University Press, 2001.

Ramazan Abacı, Nalan Kazaz, and Berkant Başa, “The Internet Addiction of Kosovo and Turkey Elderly People”, Procedia-Social and Behavioral Sciences 103 (2013) : 1104- 1117.

Roger W. Morrell,Christopher B. Mayhorn, and Joan Bennett, “A survey of World Wide Web use in middle-aged and older adults”, Human Factors 42.2 (2000) : 175-182.

Serge Proulx « Penser les usages des technologies de l'information et de la communication aujourd'hui : enjeux-modèles-tendances ", Enjeux et usages des TIC : aspects sociaux et culturels 1 (2005) : 7-20.

Sri Kurniawan, Panayiotis Zaphiris, "Research-derived web design guidelines for older people", Proceedings of the 7th international ACM SIGACCESS conference on Computers and accessibility, ACM, 2005.

Susan L.Gatto, Sunghee H. Tak, "Computer, Internet, and e-mail use among older adults : Benefits and barriers", Educational Gerontology 34.9 (2008) : 800-811.

Thompson SH Teo, Vivien KG Lim, Raye YC Lai, "Intrinsic and extrinsic motivation in Internet usage", Omega 27.1 (1999) : 25-37. 


\section{NOTES}

1. Bulletin no 24644, 16 mars 2017, http://www.tuik.gov.tr/PreHaberBultenleri.do?id=24644

2. www.tuik.gov.tr (consulté le 10 mai 2017)

3. Katz, Gurevitch et Haas (1973) offrent une typologie des besoins des utilisateurs des médias: les besoins cognitifs pour l'informa- tion, la connaissance et la compréhension de notre environnement ; besoins affectifs, pour les expériences esthétiques, agréables et émotionnelles ; besoins personnels, crédibilité, confiance, stabilité et statut personnel; besoins d'intégration sociale, le contact avec la famille, les amis et le monde ; besoins d'évasion, le détournement et la libération de tension.

4. Notre étude contient principalement trois centres : Le Centre d'éducation publique Ataşehir (Ataşehir Halk Eğitim Merkezi), le Centre d'éducation publique Kadıköy et l'École secondaire professionnelle du soir (Kadıköy Halk Eğitim Merkezi ve Akşam Sanat Okulu) et l'École du centre d'éducation publique de Kadıköy Bostancı (Kadıköy Bostancı Halk Eğitim Merkezi).

5. Quelles sont les motivations des utilisateurs et des non-utilisateurs? Combien de temps passentils sur Internet? Est-ce qu'ils possèdent un ordinateur ou une tablette? Quels sont les moyens d'accès à Internet? Que pensent-ils de l'Internet? Que signifie l'Internet? Quelles sont les difficultés ? Existe-t-il d'un soutien ou d'un empêchement de leur environne- ment social ? Après avoir appris à utiliser un ordinateur, qu'est-ce qu'ils veulent faire sur Internet? Quelles plateformes de médias sociaux connaissent-elles ou utilisent-elles? Ont-ils des comptes sociaux? Est-ce que leurs amis utilisent les réseaux sociaux?

6. La proportion de personnes âgées anal- phabètes en Turquie était de $29,2 \%$ en 2011 et devient $21,9 \%$ en 2015 (TUIK, 2017).

\section{RÉSUMÉS}

La présente étude consiste à mettre en perspective et à débattre de l'usage d'Internet par les personnes âgées à partir d'une enquête qualitative réalisée en Turquie. Ladite recherche repose sur des entretiens semi- directifs réalisés auprès de 36 personnes âgées (plus de 65 ans) vivant à Istanbul. L'échantillon de l'étude, composé de 20 utilisateurs et de 16 non-utilisateurs, englobe des entretiens qui se situent dans une approche théorique des usages et de la notion de gratification en se posant les questions de ce que font les personnes âgées avec l'Internet.

Quelles sont leurs motivations et leurs habitudes? En effet, la Turquie, à l'instar d'autres pays européens, devra faire face, dans un avenir proche, à un accroissement de la population âgée. Or, les individus composant cette démographie croissante disposent d'un certain nombre de barrières qui les empêchent de s'adapter aux besoins de la société de l'information. En exposant ainsi les difficultés d'intégrer la population âgée à l'usage de l'Internet, la présente recherche vise à souligner le risque de négligence d'une partie importante de la population dans la société d'information.

The present study consists in putting into perspective and debating the use of the Internet by the elderly from a qualitative survey conducted in Turkey. The research is based on semi-structured interviews with 
36 elderly people (over 65 years old) living in Istanbul. The study sample, composed of 20 users and

16 non-users, includes interviews that are situated in the Uses and Gratification Approach. What the elderly do with the Internet? What are their motivations and attitudes for Internet usage? Like other European countries, in Turkey an increase in the elderly population is expected in the near future. However, this growing population has barriers that prevent them from adapting to the needs of the information society and in particular to the digital age. By exposing the difficulties of integrating the elderly population into the use of the Internet, the present research aims to highlight the risk of negligence of a significant part of the population in the Information Society.

El presente estudio analiza el uso de Internet por las personas mayores y cuestiona el problema de la brecha digital a partir de una encuesta cualitativa realizada en Turquía. La investigación se basa en entrevistas semi estructuradas realizadas a 36 personas mayores (de más de 65 años) que viven en Estambul. La muestra del estudio estaba compuesta por 20 usuarios y 16 no usuarios. Las entrevistas efectuadas se sitúan dentro del enfoque teórico de los usos y la gratificación. ¿Qué hacen las personas mayores en Internet?

¿Cuáles son sus motivaciones y las razones de sus preferencias? Turquía, al igual que otros países europeos, tendrá que hacer frente a un aumento de la población de la tercera edad. Esta creciente parte de la población presenta barreras a la hora de satisfacer las necesidades de la sociedad de la información y, en particular, para adaptarse a la digitalización. Al exponer las dificultades de integrar a la población anciana en el uso de Internet, el estudio señala el riesgo de negligencia en una parte importante de la población.

INDEX

Palabras claves : Internet, personas mayores, Turquía, personas ancianos, brecha digital, tercera edad

Keywords : Internet, elderly, Turkey, digital gap

Mots-clés : Internet, personnes âgées, Turquie, fracture numérique

\section{AUTEUR}

\section{NAZLI ÜLBAY AYTUNA}

Université de Galatasaray, Istanbul, Turquie 